\title{
Utilisation du tacrolimus dans le traitement du lichen plan buccal érosif : revue de la littérature
}

Gossart R' ${ }^{1}$, Fauroux MA ${ }^{1}$, Torres $\mathrm{JH}^{1}$

1. Faculté Odontologie Montpellier - Université Montpellier I

\section{Introduction}

Le lichen plan est une dermatose inflammatoire chronique retrouvée sur la peau, les phanères et les muqueuses. Contrairement aux lésions cicatricielles, les lésions actives répondent bien au traitement conventionnel par corticothérapie. Cependant, les corticoïdes peuvent se révéler inefficaces ce qui oblige les thérapeutes à rechercher des traitements alternatifs. Le tacrolimus est un macrolide lactone dérivé de Streptomyces tsukubaensis présentant une activité immunosuppressive. En France, le tacrolimus est prescrit dans la prise en charge du rejet de greffe et des dermatites atopiques, alors qu'aux Etats-Unis il est aussi utilisé dans des cas de lichen plan buccaux symptomatiques résistants aux corticoïdes(1). Matériels et méthodes : 29 publications sur pubmed ont étudié l'utilisation du tacrolimus sous forme topique à des concentrations de $0,1 \%, 0,3 \%$ et $0,03 \%$. Les modalités vont de 2 à 3 fois par jour, et de 4 semaines à 9 mois en fonction des études.

\section{Résultats}

L'effet bénéfique du traitement a été démontré dans l'ensemble des publications. En effet, pour 25 des 28 cas traités par bain de bouche, une amélioration des lésions a pu être observée (2) (REF), de même que pour les 233 patients sur 247 traités par pommade à $0,1 \%$ (3). Une diminution des douleurs a été constatée avec la régression des lésions. Le temps de réponse du traitement a été évalué entre 1 semaine et 1 mois et demi en fonction des études. Une récidive des lésions a été observée chez 86 patients dont 4 cas 2 semaines minimum après l'arrêt du traitement. De nombreux effets secondaires ont été recensés tels que des sensations de brûlure, une sécheresse buccale, une dysgueusie, une sialorrhée ou encore une hyperpigmentation de la muqueuse, qui ont cessé avec la disparition des lésions lichénoïdes.

\section{Discussion}

Aucun cas de cancer ou d'immunodépression n'a été rapporté contrairement au risque émis. Cependant, le nombre de patients et la période de suivi semblent insuffisants pour exclure formellement le risque oncogénique du tacrolimus sous forme topique. De plus, le risque de récidive à l'arrêt du traitement requiert la prise à long terme du tacrolimus, ce qui augmente le risque d'immunodépression et de cancers secondaires potentiels.

\section{Conclusion}

Le tacrolimus dans sa forme topique pourrait donc faire officiellement partie de la pharmacopée pour l'amélioration des lésions et le soulagement des douleurs du lichen plan buccal en cas de non réponse au traitement conventionnel. Des études sur le long terme pour mieux définir les risques restent toutefois nécessaires pour une prescription en toute sécurité.

gossart.remy@gmail.com

\section{Références}

1. Al-Hashimi I et al. Oral lichen planus and oral lichenoid lesions: diagnostic and therapeutic considerations. Oral Surg Oral Med Oral Pathol Oral Radiol Endod. mars 2007;103 Suppl:S25.e1-12.

2. Rouxel A-M et al. Tacrolimus en bains de bouche dans le traitement de seconde intention du lichen plan buccal érosif. Ann Dermatol Vénéréologie. oct 2010;137(10):648-9.

3. Ribero $S$ et al. Efficacy of topical tacrolimus for oral lichen planus: real-life experience in a retrospective cohort of patients with a review of the literature. J Eur Acad Dermatol Venereol JEADV. juin 2015;29(6):1107-13.

(C) The authors, published by EDP Sciences. This is an Open Access article distributed under the terms of the Creative Commons Attribution License 4.0 (http://creativecommons.org/licenses/by/4.0/) 